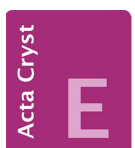

CRYSTALLOGRAPHIC COMMUNICATIONS

ISSN 2056-9890

Received 12 August 2015

Accepted 7 September 2015

Edited by H. Ishida, Okayama University, Japan

Keywords: crystal structure; cyclometalated Ru", pyrimidyl-3H-indole; para-cymene; $\mathrm{C}-\mathrm{H} \ldots \mathrm{F}$ hydrogen bonds

CCDC reference: 1027878

Supporting information: this article has supporting information at journals.iucr.org/e

\section{Crystal structure of acetonitrile $\left[\eta^{6}-1-\right.$ methyl-4-(1- methylethyl)benzene][1-(pyrimidin-2-yl)-3H-indol- 1-ium-2-yl- $\kappa^{2} N, C$ ]ruthenium(II) bis(hexafluorido- antimonate)}

\author{
Carina Sollert, ${ }^{\mathrm{a}}$ Andreas Orthaber $^{\mathrm{b}_{*}}$ and Lukasz T. Pilarski ${ }^{\mathrm{a}_{*}}$
}

${ }^{\mathbf{a}}$ Uppsala University, Department of Chemistry - BMC, Box 576, 75123 Uppsala, Sweden, and ${ }^{\mathbf{b}}$ Uppsala University, Department of Chemistry - Ångström Laboratories, Box 523, 75120 Uppsala, Sweden. *Correspondence e-mail: andreas.orthaber@kemi.uu.se, lukasz.pilarski@kemi.uu.se

In the title compound, $\left[\mathrm{Ru}\left(\mathrm{C}_{10} \mathrm{H}_{14}\right)\left(\mathrm{C}_{12} \mathrm{H}_{9} \mathrm{~N}_{3}\right)\left(\mathrm{CH}_{3} \mathrm{CN}\right)\right]\left[\mathrm{SbF}_{6}\right]_{2}$, the ruthenium(II) cation is $\eta^{6}$-coordinated by the para-cymene ligand with a $\mathrm{Ru}-$ centroid $\left(\eta^{6}\right.$-benzene) distance of 1.746 (2) A. Furthermore, ruthenium coordinations to the $\mathrm{C}$ and $\mathrm{N}$ atoms of the pyrimidyl indole ligand are found to be 1.986 (4) and 2.082 (3) $\AA$, respectively. The typical piano-stool coordination environment is saturated with an acetonitrile solvent molecule with a $\mathrm{Ru}-\mathrm{N}$ distance of 2.044 (3) $\AA$. The indolyl ligand is protonated at the C3 position with the $\mathrm{N}=\mathrm{C}$ imine bond length appropriate to that of related $3 H$-indole-based complexes. In the crystal, the complex cation is linked to the $\mathrm{SbF}_{6}{ }^{-}$ions through weak $\mathrm{C}-\mathrm{H} \cdots \mathrm{F}$ hydrogen bonds.

\section{Chemical context}

Cyclometalated ruthenium compounds are well known catalytic intermediates in the $\mathrm{C}-\mathrm{H}$ activation of various substrates (Arockiam et al., 2012; Li et al., 2012; Ferrer Flegeau et al., 2011). In a recent study on oxidative Rucatalysed heteroarene $\mathrm{C}-\mathrm{H}$ arylation (Wang et al., 2015; Ackermann \& Lygin, 2011), we demonstrated that $\left[\left\{\mathrm{RuCl}_{2}(p\right.\right.$ cymene) $\}_{2}$ ] in the presence of $\mathrm{AgSbF}_{6}$ selectively ruthenates the $\mathrm{C} 2-\mathrm{H}$ bond of $\mathrm{N}$-pyrimidine-substituted pyrroles and indoles (Sollert et al., 2015). We concluded that in our catalytic system, the resulting ruthenacyclic species likely act as precursors rather than on-cycle intermediates. In the course of our studies we observed the unusual formation of the title complex, which shows protonation at the $\mathrm{C} 3$ position. The title compound and related cyclometalated ruthenium complexes are shown schematically in Fig. 1.

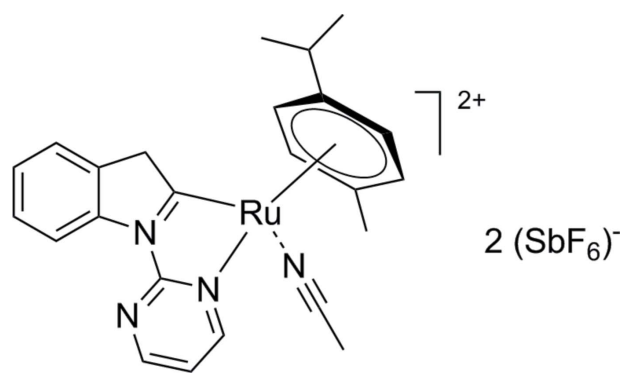

\section{Structural commentary}

In the title compound (Fig. 2), the ruthenium(II) cation is coordinated in an $\eta^{6}$ fashion by a para-cymene unit. The $\mathrm{Ru}-$ 


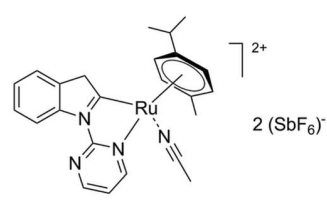

(I)

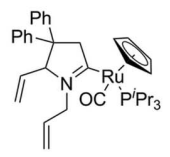

(II)

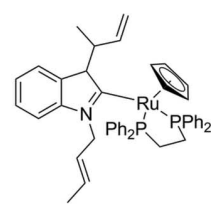

(III)
Figure 1

The title compound (I) and related cyclometalated ruthenium complexes (II) (Sollert et al., 2015) and (III) (Chiang et al., 2010).

$\mathrm{C}_{p \text {-cymene }}$ distances range from 2.197 (4) to 2.298 (4) $\AA$. The centroid of the para-cymene benzene ring $(\mathrm{Cg})$ shows an Ru1-Cg distance of 1.746 (2) $\AA$. Furthermore, ruthenium coordinations to $\mathrm{C} 2$ and $\mathrm{N} 3$ of the pyrimidyl indole are found to be 1.986 (4) and 2.082 (3) $\AA$, respectively. The coordination environment is saturated with one acetonitrile solvent molecule, with an Ru1-N5 distance of 2.044 (3) A. The leastsquares planes of the $3 H$-indole ring system [r.m.s. deviation $=$ 0.026 (4) $\AA$ ] and the pyrimidine heterocycle [r.m.s. deviation = 0.013 (4) $\AA$ ] are almost co-planar, making a dihedral angle of $2.6(2)^{\circ}$. The Ru atom deviates by only 0.056 (1) $\AA$ from the $3 H$-indole plane. The $3 H$-indole shows a clear $\mathrm{C} 2-\mathrm{N} 1$ double bond of 1.345 (5) $\AA$ in the typical range for this class of compounds. The coordinating acetonitrile solvent molecule shows slight deviation from a linear arrangement [C27 - N5$\left.\mathrm{Ru} 1=170.4(3)^{\circ}\right]$.

\section{Supramolecular features}

The packing allows no direct interaction of equivalent ruthenium complexes. The crystal packing shows a complex pattern in which two crystallographically independent $\mathrm{SbF}_{6}{ }^{-}$counter-
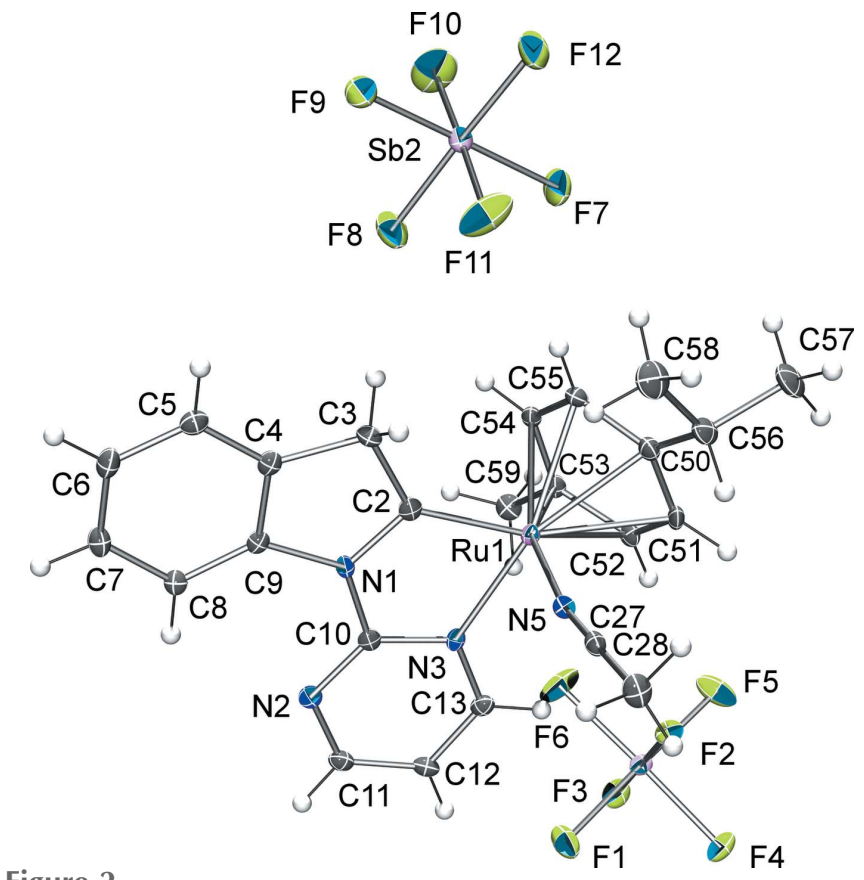

Figure 2

ORTEP representation of the molecular components of the title compound, showing $50 \%$ probability displacement ellipsoids.
Table 1

Hydrogen-bond geometry $\left(\AA{ }^{\circ}{ }^{\circ}\right)$.

\begin{tabular}{lllll}
\hline$D-\mathrm{H} \cdots A$ & $D-\mathrm{H}$ & $\mathrm{H} \cdots A$ & $D \cdots A$ & $D-\mathrm{H} \cdots A$ \\
\hline $\mathrm{C} 11-\mathrm{H} 11 \cdots \mathrm{F}^{\mathrm{i}}$ & 0.95 & 2.54 & $3.398(6)$ & 151 \\
$\mathrm{C} 12-\mathrm{H} 12 \cdots \mathrm{F} 1$ & 0.95 & 2.39 & $3.157(5)$ & 138 \\
$\mathrm{C} 13-\mathrm{H} 13 \cdots \mathrm{F} 2$ & 0.95 & 2.30 & $3.229(5)$ & 167 \\
$\mathrm{C} 51-\mathrm{H} 51 \cdots \mathrm{F} 11^{\mathrm{ii}}$ & 0.95 & 2.54 & $3.485(6)$ & 174 \\
$\mathrm{C} 52-\mathrm{H} 52 \cdots \mathrm{F} 2$ & 0.95 & 2.50 & $3.337(5)$ & 147 \\
$\mathrm{C} 54-\mathrm{H} 54 \cdots \mathrm{F} 5{ }^{\text {iii }}$ & 0.95 & 2.26 & $3.110(5)$ & 148 \\
$\mathrm{C} 59-\mathrm{H} 59 B \cdots \mathrm{F} 6$ & 0.98 & 2.32 & $3.253(6)$ & 158 \\
$\mathrm{C} 59-\mathrm{H} 59 C \cdots \mathrm{F} 5^{\text {iii }}$ & 0.98 & 2.45 & $3.270(6)$ & 141
\end{tabular}

Symmetry codes: (i) $x,-y+\frac{1}{2}, z+\frac{1}{2}$; (ii) $-x+2, y-\frac{1}{2},-z+\frac{3}{2}$; (iii) $-x+\frac{3}{2}, y+\frac{1}{2}, z$.

ions occupy a void formed by symmetry-equivalent metal complexes. $\mathrm{C}-\mathrm{H}$ hydrogen bonds of the pyrimidylindole and para-cymene ligands with the $\mathrm{SbF}_{6}{ }^{-}$ions mainly account for the observed packing pattern (Table 1).

\section{Database survey}

This structure is related to chloro $\left(\eta^{6}\right.$-para-cymene $)\left[\kappa^{2}-N, C-1-\right.$ (pyrimidin-2-yl)-1H-indole]ruthenium (Sollert et al., 2015), in which the double bond is at $\mathrm{C} 2=\mathrm{C} 3$. The Ru1-C2 and Ru1cymene distances, however, are almost unaltered. This is consistent with the development of a positive charge at N1 to effect the $\mathrm{C} 3$ protonation rather than at the $\mathrm{Ru}^{\mathrm{II}}$ atom. The $\mathrm{C} 2$ atom in the title compound is therefore formally an anionic ligand, and not a carbene carbon. A similar cyclometalated pyrrolinyl complex (2) Buil et al., 2015; Fig. 1) was obtained through $\mathrm{HBF}_{4}$-mediated rearrangement of $N$-allylic substituents. The Ru-C distances of 2.077 (4) $\AA$ (Buil et al., 2003) are comparable to the $\mathrm{Ru} 1-\mathrm{C} 2$ distance of the title compound. The Ru-catalysed rearrangement of a 1,7-eneyne afforded the $\mathrm{C} 2$-cyclometalated $3 \mathrm{H}$-indole (3) (Chiang et al., 2010; Fig. 1). Structural parameters of this cyclopentadienylcoordinated ruthenium complex are in good agreement with the title compound.

\section{Synthesis and crystallization}

A pre-dried Young's tube was charged with chlorido $\left(\eta^{6}\right.$-paracymene) $\left[\kappa^{2}-N, C-1-(\right.$ pyrimidin-2-yl)- $1 H$-indole $]$ ruthenium (50 mg, 1.0 equiv., $0.11 \mathrm{mmol}$ ) and $\mathrm{AgSbF}_{6}$ (76 mg, 2.0 equiv., $0.22 \mathrm{mmol}$ ). The tube was evacuated and backfilled with argon three times. The tube was equipped with a rubber septum and anhydrous MeCN ( $2 \mathrm{~mL}$ ) was added via a syringe. The septum was removed, the tube sealed and wrapped in aluminium foil to protect the reaction mixture from light. The mixture was left stirring at room temperature for $18 \mathrm{~h}$, after which the resulting precipitate was filtered off rapidly under air and the filtrate transferred immediately into a pre-dried roundbottom flask under argon. The solvent was evaporated under reduced pressure and a green solid was obtained. The solid was dissolved in $\mathrm{d}_{8}$-THF and transferred into a NMR tube under argon. The title compound was obtained as green crystals upon slow evaporation of the solvent. 
Table 2

Experimental details.

\begin{tabular}{|c|c|}
\hline \multicolumn{2}{|l|}{ Crystal data } \\
\hline Chemical formula & $\begin{array}{l}{\left[\mathrm{Ru}\left(\mathrm{C}_{10} \mathrm{H}_{14}\right)\left(\mathrm{C}_{12} \mathrm{H}_{9} \mathrm{~N}_{3}\right)\left(\mathrm{C}_{2} \mathrm{H}_{3} \mathrm{~N}\right)\right]-} \\
{\left[\mathrm{SbF}_{6}\right]_{2}}\end{array}$ \\
\hline$M_{\mathrm{r}}$ & 943.06 \\
\hline Crystal system, space group & Orthorhombic, $\mathrm{Pbca}$ \\
\hline Temperature $(\mathrm{K})$ & 100 \\
\hline$a, b, c(\AA)$ & $\begin{array}{l}16.6046(8), 15.5955(7), \\
\quad 23.2786(12)\end{array}$ \\
\hline$V\left(\AA^{3}\right)$ & $6028.2(5)$ \\
\hline$Z$ & 8 \\
\hline Radiation type & Мо $K \alpha$ \\
\hline$\mu\left(\mathrm{mm}^{-1}\right)$ & 2.37 \\
\hline Crystal size $(\mathrm{mm})$ & $0.18 \times 0.17 \times 0.08$ \\
\hline \multicolumn{2}{|l|}{ Data collection } \\
\hline Diffractometer & BrukerAPEXII with CCD \\
\hline Absorption correction & $\begin{array}{l}\text { Multi-scan (SADABS; Sheldrick, } \\
\text { 1996) }\end{array}$ \\
\hline$T_{\min }, T_{\max }$ & $0.578,0.746$ \\
\hline $\begin{array}{l}\text { No. of measured, independent and } \\
\text { observed }[I>2 \sigma(I)] \text { reflections }\end{array}$ & $27645,6643,4920$ \\
\hline$R_{\text {int }}$ & 0.054 \\
\hline$(\sin \theta / \lambda)_{\max }\left(\AA^{-1}\right)$ & 0.643 \\
\hline \multicolumn{2}{|l|}{ Refinement } \\
\hline$R\left[F^{2}>2 \sigma\left(F^{2}\right)\right], w R\left(F^{2}\right), S$ & $0.033,0.074,1.01$ \\
\hline No. of reflections & 6643 \\
\hline No. of parameters & 392 \\
\hline $\mathrm{H}$-atom treatment & H-atom parameters constrained \\
\hline$\Delta \rho_{\max }, \Delta \rho_{\min }\left(\mathrm{e} \AA^{-3}\right)$ & $0.89,-1.00$ \\
\hline
\end{tabular}

Computer programs: APEX2 and SAINT (Bruker, 2012), SHELXS97 (Sheldrick, 2008), SHELXL2014 (Sheldrick, 2015) and ORTEP-3 for Windows (Farrugia, 2012).

\section{Refinement}

Crystal data, data collection and refinement details are summarized in Table 2. All $\mathrm{H}$ atoms on carbon were placed at calculated positions $[\mathrm{C}-\mathrm{H}=0.95$ (aromatic), 0.98 (methyl), 0.99 (methylene) and 1.00 (methine) $\AA$ ] using a riding model with $U_{\text {iso }}(\mathrm{H})=1.2 U_{\text {eq }}(\mathrm{C})$ or $1.5 U_{\text {eq }}\left(\mathrm{C}_{\text {methyl }}\right)$. The $\mathrm{Ru}-\mathrm{C}$ bonds were ignored in the ideal placement of the aromatic $\mathrm{H}$ atoms.

\section{Acknowledgements}

The authors would like to thank the Swedish research council (Vetenskapsrådet) for support.

\section{References}

Ackermann, L. \& Lygin, A. V. (2011). Org. Lett. 13, 3332-3335.

Arockiam, P. B., Bruneau, C. \& Dixneuf, P. H. (2012). Chem. Rev. 112, $5879-5918$.

Bruker (2012). APEX2 and SAINT. Bruker AXS Inc. Madison, Wisconsin, USA.

Buil, M. L., Esteruelas, M. A., López, A. M. \& Oñate, E. (2003). Organometallics, 22, 5274-5284.

Chiang, P.-Y., Lin, Y.-C., Wang, Y. \& Liu, Y.-H. (2010). Organometallics, 29, 5776-5782.

Farrugia, L. J. (2012). J. Appl. Cryst. 45, 849-854.

Ferrer Flegeau, E., Bruneau, C., Dixneuf, P. H. \& Jutand, A. (2011). J. Am. Chem. Soc. 133, 10161-10170.

Li, B., Roisnel, T., Darcel, C. \& Dixneuf, P. H. (2012). Dalton Trans. 41, 10934-10937.

Sheldrick, G. M. (1996). SADABS. University of Göttingen, Germany.

Sheldrick, G. M. (2008). Acta Cryst. A64, 112-122.

Sheldrick, G. M. (2015). Acta Cryst. C71, 3-8.

Sollert, C., Devaraj, K., Orthaber, A., Gates, P. J. \& Pilarski, L. T. (2015). Chem. Eur. J. 21, 5380-5386.

Wang, L., Yang, D., Han, F., Li, D., Zhao, D. \& Wang, R. (2015). Org. Lett. 17, 176-179. 


\section{supporting information}

Acta Cryst. (2015). E71, 1190-1192［doi:10.1107/S2056989015016710]

\section{Crystal structure of acetonitrile[ $\eta^{6}-1$-methyl-4-(1-methylethyl)benzene] [1-(pyrimidin-2-yl)-3H-indol-1-ium-2-yl- $\left.\kappa^{2} N, C\right]$ ruthenium(II) bis- (hexafluoridoantimonate)}

\section{Carina Sollert, Andreas Orthaber and Lukasz T. Pilarski}

\section{Computing details}

Data collection: APEX2 (Bruker, 2012); cell refinement: SAINT (Bruker, 2012); data reduction: SAINT (Bruker, 2012); program(s) used to solve structure: SHELXS97 (Sheldrick, 2008); program(s) used to refine structure: SHELXL2014 (Sheldrick, 2015); molecular graphics: ORTEP-3 for Windows (Farrugia, 2012); software used to prepare material for publication: SHELXL2014 (Sheldrick, 2015).

Acetonitrile[ $\eta^{6}$-1-methyl-4-(1-methylethyl)benzene][1-(pyrimidin-2-yl)-3H-indol-1-ium-2-yl- $\left.\kappa^{2} N, C\right]$ ruthenium(II) bis(hexafluoridoantimonate)

Crystal data

$\left[\mathrm{Ru}\left(\mathrm{C}_{10} \mathrm{H}_{14}\right)\left(\mathrm{C}_{12} \mathrm{H}_{9} \mathrm{~N}_{3}\right)\left(\mathrm{C}_{2} \mathrm{H}_{3} \mathrm{~N}\right)\right]\left[\mathrm{SbF}_{6}\right]_{2}$

$M_{r}=943.06$

Orthorhombic, $\mathrm{Pbca}$

$a=16.6046(8) \AA$

$b=15.5955$ (7) $\AA$

$c=23.2786(12) \AA$

$V=6028.2(5) \AA^{3}$

$Z=8$

$F(000)=3616$

Data collection

BrukerAPEXII with CCD

diffractometer

Radiation source: fine-focus sealed tube

Graphite monochromator

$\omega$ scans

Absorption correction: multi-scan

(SADABS; Sheldrick, 1996)

$T_{\min }=0.578, T_{\max }=0.746$

Refinement

Refinement on $F^{2}$

Least-squares matrix: full

$R\left[F^{2}>2 \sigma\left(F^{2}\right)\right]=0.033$

$w R\left(F^{2}\right)=0.074$

$S=1.01$

6643 reflections
$D_{\mathrm{x}}=2.078 \mathrm{Mg} \mathrm{m}^{-3}$

Mo $K \alpha$ radiation, $\lambda=0.71073 \AA$

Cell parameters from 4920 reflections

$\theta=1.8-25.2^{\circ}$

$\mu=2.37 \mathrm{~mm}^{-1}$

$T=100 \mathrm{~K}$

Plate, green

$0.18 \times 0.17 \times 0.08 \mathrm{~mm}$

27645 measured reflections

6643 independent reflections

4920 reflections with $I>2 \sigma(I)$

$R_{\text {int }}=0.054$

$\theta_{\max }=27.2^{\circ}, \theta_{\min }=1.8^{\circ}$

$h=-21 \rightarrow 20$

$k=-19 \rightarrow 19$

$l=-29 \rightarrow 27$

392 parameters

0 restraints

Hydrogen site location: inferred from neighbouring sites

$\mathrm{H}$-atom parameters constrained 
$w=1 /\left[\sigma^{2}\left(F_{\mathrm{o}}^{2}\right)+(0.0294 P)^{2}+2.8958 P\right]$

where $P=\left(F_{\mathrm{o}}^{2}+2 F_{\mathrm{c}}^{2}\right) / 3$

$(\Delta / \sigma)_{\max }=0.002$

$$
\Delta \rho_{\max }=0.89 \text { e } \AA^{-3}
$$$$
\Delta \rho_{\min }=-1.00 \mathrm{e} \AA^{-3}
$$

\section{Special details}

Experimental. X-ray crystallographic data for I were collected from a single-crystal sample, which was mounted on a loop fiber. Data were collected using a Bruker smart diffractometer equipped with an APEX II CCD Detector, a graphite monochromator. The crystal-to-detector distance was $5.0 \mathrm{~cm}$, and the data collection was carried out in $512 \times 512$ pixel mode.

Geometry. All e.s.d.'s (except the e.s.d. in the dihedral angle between two 1.s. planes) are estimated using the full covariance matrix. The cell e.s.d.'s are taken into account individually in the estimation of e.s.d.'s in distances, angles and torsion angles; correlations between e.s.d.'s in cell parameters are only used when they are defined by crystal symmetry. An approximate (isotropic) treatment of cell e.s.d.'s is used for estimating e.s.d.'s involving 1.s. planes.

Refinement. Refinement of $F^{2}$ against ALL reflections. The weighted $R$-factor $w R$ and goodness of fit $S$ are based on $F^{2}$, conventional $R$-factors $R$ are based on $F$, with $F$ set to zero for negative $F^{2}$. The threshold expression of $F^{2}>\sigma\left(F^{2}\right)$ is used only for calculating $R$-factors(gt) etc. and is not relevant to the choice of reflections for refinement. $R$-factors based on $F^{2}$ are statistically about twice as large as those based on $F$, and $R$ - factors based on ALL data will be even larger.

Fractional atomic coordinates and isotropic or equivalent isotropic displacement parameters $\left(\AA^{2}\right)$

\begin{tabular}{|c|c|c|c|c|}
\hline & $x$ & $y$ & $z$ & $U_{\text {iso }} * / U_{\text {eq }}$ \\
\hline Ru1 & $0.93384(2)$ & $0.22549(2)$ & $0.87058(2)$ & $0.01738(8)$ \\
\hline $\mathrm{Sb} 1$ & $0.85442(2)$ & $-0.13417(2)$ & $0.94657(2)$ & $0.02309(8)$ \\
\hline $\mathrm{Sb} 2$ & $0.78888(2)$ & $0.49530(2)$ & $0.71378(2)$ & $0.02768(8)$ \\
\hline F1 & $0.89213(19)$ & $-0.09995(16)$ & $1.01877(12)$ & $0.0461(8)$ \\
\hline $\mathrm{F} 2$ & $0.93350(14)$ & $-0.06576(14)$ & $0.91106(11)$ & $0.0282(6)$ \\
\hline F3 & $0.77479(15)$ & $-0.20299(16)$ & $0.98028(12)$ & $0.0372(7)$ \\
\hline $\mathrm{F} 4$ & $0.92598(15)$ & $-0.22620(14)$ & $0.94954(12)$ & $0.0359(6)$ \\
\hline F5 & $0.82004(18)$ & $-0.1684(2)$ & $0.87420(12)$ & $0.0517(8)$ \\
\hline F6 & $0.78500(18)$ & $-0.04062(18)$ & $0.94558(15)$ & $0.0605(10)$ \\
\hline F7 & $0.7883(2)$ & $0.37969(16)$ & $0.69278(13)$ & $0.0558(9)$ \\
\hline F8 & $0.7572(2)$ & $0.46434(18)$ & $0.78733(12)$ & $0.0538(9)$ \\
\hline F9 & $0.79200(19)$ & $0.61114(16)$ & $0.73516(12)$ & $0.0464(8)$ \\
\hline F10 & $0.6831(2)$ & $0.5081(2)$ & 0.69167 (18) & 0.0795 (12) \\
\hline F11 & 0.89525 (19) & 0.48324 (19) & $0.73806(18)$ & $0.0732(11)$ \\
\hline F12 & $0.8239(3)$ & $0.5243(2)$ & $0.64075(14)$ & $0.0872(14)$ \\
\hline N1 & 0.90603 (19) & $0.3372(2)$ & $0.96626(14)$ & $0.0178(7)$ \\
\hline N2 & $0.8897(2)$ & $0.2522(2)$ & $1.04905(14)$ & $0.0214(8)$ \\
\hline N3 & $0.91640(18)$ & $0.1923(2)$ & $0.95632(14)$ & $0.0176(7)$ \\
\hline N5 & $1.0551(2)$ & $0.2291(2)$ & $0.88533(14)$ & $0.0210(8)$ \\
\hline $\mathrm{C} 2$ & $0.9204(2)$ & $0.3380(3)$ & $0.90940(18)$ & $0.0206(9)$ \\
\hline $\mathrm{C} 3$ & $0.9232(3)$ & $0.4290(2)$ & 0.89077 (17) & $0.0223(9)$ \\
\hline $\mathrm{H} 3 \mathrm{~A}$ & 0.8806 & 0.4410 & 0.8621 & $0.027 *$ \\
\hline H3B & 0.9762 & 0.4431 & 0.8738 & $0.027 *$ \\
\hline $\mathrm{C} 4$ & $0.9091(2)$ & $0.4796(2)$ & $0.94537(18)$ & $0.0214(9)$ \\
\hline $\mathrm{C} 5$ & $0.9052(3)$ & $0.5662(3)$ & 0.95639 (19) & $0.0263(10)$ \\
\hline H5 & 0.9146 & 0.6068 & 0.9267 & $0.032 *$ \\
\hline C6 & $0.8872(3)$ & $0.5931(3)$ & $1.0116(2)$ & $0.0281(10)$ \\
\hline H6 & 0.8847 & 0.6528 & 1.0196 & $0.034^{*}$ \\
\hline
\end{tabular}




\begin{tabular}{|c|c|c|c|c|}
\hline C7 & $0.8729(3)$ & $0.5347(3)$ & $1.05571(19)$ & $0.0267(10)$ \\
\hline $\mathrm{H} 7$ & 0.8592 & 0.5549 & 1.0930 & $0.032 *$ \\
\hline $\mathrm{C} 8$ & $0.8786(2)$ & $0.4467(3)$ & $1.04542(18)$ & $0.0223(9)$ \\
\hline $\mathrm{H} 8$ & 0.8703 & 0.4058 & 1.0751 & $0.027 *$ \\
\hline C9 & 0.8969 (2) & $0.4222(2)$ & $0.99016(18)$ & $0.0191(9)$ \\
\hline $\mathrm{C} 10$ & $0.9032(2)$ & $0.2573(2)$ & $0.99352(18)$ & $0.0190(9)$ \\
\hline C11 & $0.8882(2)$ & $0.1724(3)$ & $1.07022(18)$ & $0.0230(9)$ \\
\hline H11 & 0.8788 & 0.1650 & 1.1102 & $0.028 *$ \\
\hline $\mathrm{C} 12$ & $0.8997(2)$ & $0.1006(3)$ & 1.03687 (17) & $0.0229(9)$ \\
\hline H12 & 0.8967 & 0.0446 & 1.0527 & $0.028 *$ \\
\hline $\mathrm{C} 13$ & $0.9157(2)$ & $0.1136(2)$ & $0.97918(18)$ & $0.0219(9)$ \\
\hline H13 & 0.9264 & 0.0655 & 0.9553 & $0.026^{*}$ \\
\hline $\mathrm{C} 27$ & $1.1233(3)$ & $0.2267(2)$ & 0.88601 (17) & $0.0209(9)$ \\
\hline $\mathrm{C} 28$ & $1.2103(3)$ & $0.2249(3)$ & $0.8860(2)$ & $0.0326(11)$ \\
\hline $\mathrm{H} 28 \mathrm{~A}$ & 1.2303 & 0.2392 & 0.8476 & $0.049^{*}$ \\
\hline $\mathrm{H} 28 \mathrm{~B}$ & 1.2289 & 0.1674 & 0.8967 & $0.049^{*}$ \\
\hline $\mathrm{H} 28 \mathrm{C}$ & 1.2307 & 0.2668 & 0.9138 & $0.049^{*}$ \\
\hline $\mathrm{C} 50$ & $0.9606(3)$ & $0.2124(3)$ & $0.77680(18)$ & $0.0247(10)$ \\
\hline C51 & $0.9519(3)$ & $0.1264(3)$ & $0.79863(17)$ & $0.0241(10)$ \\
\hline H51 & 0.9932 & 0.0856 & 0.7916 & $0.029^{*}$ \\
\hline C52 & $0.8850(3)$ & $0.1023(3)$ & $0.82936(17)$ & $0.0227(9)$ \\
\hline H52 & 0.8808 & 0.0452 & 0.8433 & $0.027 *$ \\
\hline $\mathrm{C} 53$ & $0.8216(2)$ & $0.1625(3)$ & $0.84050(18)$ & $0.0207(9)$ \\
\hline C54 & $0.8274(2)$ & $0.2446(2)$ & $0.81594(16)$ & $0.0196(9)$ \\
\hline H54 & 0.7842 & 0.2840 & 0.8205 & $0.024 *$ \\
\hline C55 & $0.8962(2)$ & 0.2697 (3) & 0.78465 (17) & $0.0213(9)$ \\
\hline H55 & 0.8990 & 0.3257 & 0.7687 & $0.026^{*}$ \\
\hline C56 & $1.0360(3)$ & $0.2354(3)$ & $0.74384(19)$ & $0.0305(11)$ \\
\hline H56 & 1.0823 & 0.2063 & 0.7631 & $0.037^{*}$ \\
\hline C57 & $1.0281(3)$ & 0.1975 (3) & $0.6834(2)$ & $0.0448(14)$ \\
\hline H57A & 1.0189 & 0.1355 & 0.6861 & $0.067 *$ \\
\hline H57B & 1.0777 & 0.2083 & 0.6617 & $0.067^{*}$ \\
\hline $\mathrm{H} 57 \mathrm{C}$ & 0.9825 & 0.2244 & 0.6635 & $0.067^{*}$ \\
\hline C58 & $1.0541(3)$ & $0.3316(3)$ & $0.7425(2)$ & $0.0445(13)$ \\
\hline $\mathrm{H} 58 \mathrm{~A}$ & 1.1071 & 0.3411 & 0.7252 & $0.067^{*}$ \\
\hline $\mathrm{H} 58 \mathrm{C}$ & 1.0539 & 0.3542 & 0.7818 & $0.067^{*}$ \\
\hline H58B & 1.0129 & 0.3611 & 0.7198 & $0.067 *$ \\
\hline C59 & $0.7499(3)$ & $0.1355(3)$ & $0.87511(19)$ & $0.0283(10)$ \\
\hline $\mathrm{H} 59 \mathrm{C}$ & 0.7273 & 0.1855 & 0.8949 & $0.042 *$ \\
\hline H59B & 0.7664 & 0.0926 & 0.9035 & $0.042^{*}$ \\
\hline H59A & 0.7091 & 0.1108 & 0.8496 & $0.042 *$ \\
\hline
\end{tabular}

Atomic displacement parameters $\left(\AA^{2}\right)$

\begin{tabular}{lllllll}
\hline & $U^{11}$ & $U^{22}$ & $U^{33}$ & $U^{12}$ & $U^{13}$ & $U^{23}$ \\
\hline Ru1 & $0.01528(16)$ & $0.01805(16)$ & $0.01879(17)$ & $0.00165(13)$ & $0.00117(14)$ & $-0.00097(14)$ \\
Sb1 & $0.01982(15)$ & $0.02391(15)$ & $0.02554(16)$ & $0.00207(12)$ & $0.00044(13)$ & $0.00291(13)$ \\
Sb2 & $0.03160(17)$ & $0.02741(16)$ & $0.02404(16)$ & $0.00270(13)$ & $0.00236(13)$ & $-0.00066(13)$
\end{tabular}


supporting information

\begin{tabular}{|c|c|c|c|c|c|c|}
\hline $\mathrm{F} 1$ & $0.073(2)$ & $0.0358(15)$ & $0.0295(16)$ & $-0.0094(15)$ & $0.0018(15)$ & $-0.0053(13)$ \\
\hline F2 & $0.0254(13)$ & $0.0237(12)$ & $0.0354(15)$ & $-0.0027(11)$ & $-0.0018(12)$ & $0.0042(11)$ \\
\hline F3 & $0.0258(15)$ & $0.0391(15)$ & $0.0466(17)$ & $-0.0006(12)$ & $0.0117(13)$ & $0.0062(13)$ \\
\hline F4 & $0.0288(15)$ & $0.0234(13)$ & $0.0557(18)$ & $0.0042(11)$ & $0.0071(13)$ & $0.0046(13)$ \\
\hline F5 & 0.0488 (19) & $0.075(2)$ & $0.0315(17)$ & $-0.0302(17)$ & $-0.0106(14)$ & $0.0072(15)$ \\
\hline F6 & $0.0386(18)$ & $0.0464(17)$ & $0.096(3)$ & $0.0217(14)$ & $0.0235(18)$ & $0.0285(18)$ \\
\hline F7 & $0.089(3)$ & $0.0309(16)$ & 0.0481 (19) & $0.0035(15)$ & $0.0041(18)$ & $-0.0131(14)$ \\
\hline F8 & $0.082(2)$ & $0.0438(17)$ & $0.0351(17)$ & $-0.0126(17)$ & $0.0162(16)$ & $0.0004(14)$ \\
\hline F9 & $0.068(2)$ & $0.0297(15)$ & $0.0410(17)$ & $-0.0018(14)$ & $0.0026(16)$ & $0.0015(13)$ \\
\hline F10 & $0.055(2)$ & $0.085(3)$ & $0.098(3)$ & $0.011(2)$ & $-0.041(2)$ & $-0.017(2)$ \\
\hline F11 & $0.0317(18)$ & $0.0531(19)$ & $0.135(4)$ & $0.0016(15)$ & -0.007 (2) & $0.019(2)$ \\
\hline F12 & $0.164(4)$ & $0.055(2)$ & $0.042(2)$ & $0.028(2)$ & $0.048(2)$ & $0.0118(17)$ \\
\hline N1 & $0.0178(17)$ & $0.0198(17)$ & $0.0158(18)$ & $0.0006(14)$ & $0.0008(14)$ & $-0.0036(14)$ \\
\hline $\mathrm{N} 2$ & $0.0171(18)$ & $0.0243(19)$ & $0.023(2)$ & $0.0003(15)$ & $-0.0010(15)$ & $-0.0002(16)$ \\
\hline N3 & $0.0131(17)$ & $0.0188(17)$ & 0.0209 (19) & $0.0016(13)$ & $0.0025(14)$ & $-0.0020(15)$ \\
\hline N5 & $0.023(2)$ & $0.0222(18)$ & $0.0182(19)$ & $0.0017(15)$ & $0.0006(15)$ & $-0.0003(15)$ \\
\hline $\mathrm{C} 2$ & $0.010(2)$ & $0.027(2)$ & $0.024(2)$ & $0.0011(16)$ & $-0.0018(17)$ & $-0.0022(18)$ \\
\hline C3 & $0.025(2)$ & $0.025(2)$ & 0.017 (2) & $-0.0011(18)$ & $0.0005(18)$ & 0.0039 (18) \\
\hline $\mathrm{C} 4$ & $0.014(2)$ & $0.023(2)$ & $0.027(2)$ & $0.0000(16)$ & $-0.0017(18)$ & $-0.0017(19)$ \\
\hline $\mathrm{C} 5$ & $0.022(2)$ & $0.027(2)$ & $0.030(3)$ & $0.0025(18)$ & $-0.0022(19)$ & $0.003(2)$ \\
\hline C6 & $0.024(2)$ & $0.025(2)$ & $0.035(3)$ & $0.0022(19)$ & $-0.002(2)$ & $-0.009(2)$ \\
\hline $\mathrm{C} 7$ & $0.025(2)$ & $0.028(2)$ & $0.027(2)$ & $-0.0029(19)$ & $0.002(2)$ & $-0.008(2)$ \\
\hline C8 & $0.020(2)$ & $0.022(2)$ & $0.024(2)$ & $-0.0023(17)$ & $0.0005(18)$ & $-0.0019(19)$ \\
\hline $\mathrm{C} 9$ & $0.013(2)$ & $0.019(2)$ & $0.025(2)$ & $-0.0008(16)$ & $-0.0015(17)$ & $-0.0016(18)$ \\
\hline $\mathrm{C} 10$ & 0.0097 (19) & $0.025(2)$ & $0.022(2)$ & $-0.0012(16)$ & $-0.0028(17)$ & $-0.0017(18)$ \\
\hline C11 & $0.020(2)$ & $0.030(2)$ & $0.020(2)$ & $0.0007(18)$ & $-0.0028(18)$ & 0.0031 (19) \\
\hline $\mathrm{C} 12$ & $0.022(2)$ & $0.027(2)$ & $0.020(2)$ & $-0.0031(18)$ & $-0.0039(18)$ & $0.0056(19)$ \\
\hline C13 & $0.020(2)$ & $0.020(2)$ & $0.026(2)$ & $0.0012(16)$ & $-0.0060(19)$ & $-0.0006(18)$ \\
\hline $\mathrm{C} 27$ & $0.026(2)$ & $0.020(2)$ & $0.017(2)$ & $-0.0017(18)$ & $0.0019(18)$ & $-0.0029(17)$ \\
\hline $\mathrm{C} 28$ & $0.020(2)$ & $0.043(3)$ & 0.035 & $-0.005(2)$ & $0.002(2)$ & $-0.007(2)$ \\
\hline C50 & $0.024(2)$ & $0.032(2)$ & $0.018(2)$ & $0.0057(19)$ & $-0.0021(18)$ & $-0.0047(19)$ \\
\hline C51 & $0.026(2)$ & $0.027(2)$ & $0.019(2)$ & $0.0100(18)$ & $-0.0035(18)$ & $-0.0101(19)$ \\
\hline C52 & $0.027(2)$ & $0.022(2)$ & $0.020(2)$ & $-0.0010(18)$ & $-0.0037(19)$ & $-0.0070(18)$ \\
\hline C53 & $0.015(2)$ & $0.025(2)$ & $0.022(2)$ & 0.0001 (17) & $-0.0028(18)$ & $-0.0043(18)$ \\
\hline C54 & $0.016(2)$ & 0.025 & $0.017(2)$ & $0.0041(17)$ & $-0.0047(17)$ & $-0.0043(18)$ \\
\hline C55 & $0.024(2)$ & $0.024(2)$ & $0.016(2)$ & $0.0022(18)$ & $-0.0005(18)$ & $0.0024(18)$ \\
\hline C56 & $0.026(2)$ & $0.043(3)$ & $0.022(2)$ & $0.005(2)$ & $0.007(2)$ & $0.003(2)$ \\
\hline C57 & 0.045 & $0.066(4)$ & $0.023(3)$ & $0.003(3)$ & $0.014(2)$ & 0.001 \\
\hline C58 & $0.036(3)$ & $0.053(3)$ & $0.045(3)$ & $-0.004(3)$ & $0.013(3)$ & $0.001(3)$ \\
\hline C59 & $0.026(2)$ & $0.028(2)$ & $0.031(3)$ & $-0.006(2)$ & $-0.001(2)$ & $-0.001(2)$ \\
\hline
\end{tabular}

Geometric parameters (A, $\stackrel{\circ}{)}$

\begin{tabular}{llll}
\hline Ru1-C2 & $1.986(4)$ & C56-C57 & $1.533(6)$ \\
Ru1-N3 & $2.082(3)$ & C56-H56 & 1.0000 \\
Ru1-N5 & $2.044(3)$ & C57-H57A & 0.9800 \\
Ru1-C50 & $2.237(4)$ & C57-H57B & 0.9800 \\
Ru1-C51 & $2.298(4)$ & C57-H57C & 0.9800
\end{tabular}




\begin{tabular}{|c|c|c|c|}
\hline Ru1-C52 & $2.296(4)$ & C58-H58A & 0.9800 \\
\hline Ru1-C53 & $2.220(4)$ & $\mathrm{C} 58-\mathrm{H} 58 \mathrm{C}$ & 0.9800 \\
\hline Ru1-C54 & $2.197(4)$ & C58-H58B & 0.9800 \\
\hline Ru1-C55 & $2.206(4)$ & $\mathrm{C} 2-\mathrm{N} 1$ & $1.345(5)$ \\
\hline $\mathrm{Sb} 1-\mathrm{F} 1$ & $1.871(3)$ & $\mathrm{C} 2-\mathrm{C} 3$ & $1.485(5)$ \\
\hline $\mathrm{Sb} 1-\mathrm{F} 2$ & $1.883(2)$ & $\mathrm{C} 3-\mathrm{C} 4$ & $1.514(5)$ \\
\hline $\mathrm{Sb} 1-\mathrm{F} 3$ & $1.875(2)$ & $\mathrm{C} 3-\mathrm{H} 3 \mathrm{~A}$ & 0.9900 \\
\hline $\mathrm{Sb} 1-\mathrm{F} 4$ & $1.865(2)$ & $\mathrm{C} 3-\mathrm{H} 3 \mathrm{~B}$ & 0.9900 \\
\hline $\mathrm{Sb} 1-\mathrm{F} 5$ & $1.857(3)$ & $\mathrm{C} 4-\mathrm{C} 5$ & $1.377(6)$ \\
\hline $\mathrm{Sb} 1-\mathrm{F} 6$ & $1.860(3)$ & $\mathrm{C} 4-\mathrm{C} 9$ & $1.388(5)$ \\
\hline $\mathrm{Sb} 2-\mathrm{F} 7$ & $1.868(3)$ & $\mathrm{C} 9-\mathrm{C} 8$ & $1.376(5)$ \\
\hline $\mathrm{Sb} 2-\mathrm{F} 8$ & $1.855(3)$ & $\mathrm{C} 9-\mathrm{N} 1$ & $1.447(5)$ \\
\hline $\mathrm{Sb} 2-\mathrm{F} 9$ & $1.875(3)$ & $\mathrm{C} 8-\mathrm{C} 7$ & $1.396(6)$ \\
\hline $\mathrm{Sb} 2-\mathrm{F} 10$ & $1.841(3)$ & $\mathrm{C} 8-\mathrm{H} 8$ & 0.9500 \\
\hline $\mathrm{Sb} 2-\mathrm{F} 11$ & $1.864(3)$ & $\mathrm{C} 7-\mathrm{C} 6$ & $1.393(6)$ \\
\hline $\mathrm{Sb} 2-\mathrm{F} 12$ & $1.853(3)$ & $\mathrm{C} 7-\mathrm{H} 7$ & 0.9500 \\
\hline N5-C27 & $1.133(5)$ & $\mathrm{C} 6-\mathrm{C} 5$ & $1.384(6)$ \\
\hline $\mathrm{C} 50-\mathrm{C} 55$ & $1.406(6)$ & $\mathrm{C} 6-\mathrm{H} 6$ & 0.9500 \\
\hline $\mathrm{C} 50-\mathrm{C} 51$ & $1.442(6)$ & $\mathrm{C} 5-\mathrm{H} 5$ & 0.9500 \\
\hline $\mathrm{C} 50-\mathrm{C} 56$ & $1.510(6)$ & $\mathrm{N} 1-\mathrm{C} 10$ & $1.399(5)$ \\
\hline C51-C52 & $1.373(6)$ & $\mathrm{C} 10-\mathrm{N} 2$ & $1.314(5)$ \\
\hline C51-H51 & 0.9500 & $\mathrm{C} 10-\mathrm{N} 3$ & $1.351(5)$ \\
\hline $\mathrm{C} 52-\mathrm{C} 53$ & $1.436(5)$ & N2-C11 & $1.339(5)$ \\
\hline C52-H52 & 0.9500 & $\mathrm{C} 11-\mathrm{C} 12$ & $1.375(6)$ \\
\hline $\mathrm{C} 53-\mathrm{C} 54$ & $1.406(5)$ & $\mathrm{C} 11-\mathrm{H} 11$ & 0.9500 \\
\hline C53-C59 & $1.497(6)$ & $\mathrm{C} 12-\mathrm{C} 13$ & $1.384(6)$ \\
\hline C54-C55 & $1.409(6)$ & $\mathrm{C} 12-\mathrm{H} 12$ & 0.9500 \\
\hline C54-H54 & 0.9500 & $\mathrm{C} 13-\mathrm{N} 3$ & $1.338(5)$ \\
\hline $\mathrm{C} 55-\mathrm{H} 55$ & 0.9500 & $\mathrm{C} 13-\mathrm{H} 13$ & 0.9500 \\
\hline C59-H59C & 0.9800 & $\mathrm{C} 27-\mathrm{C} 28$ & $1.444(6)$ \\
\hline С59-H59B & 0.9800 & $\mathrm{C} 28-\mathrm{H} 28 \mathrm{~A}$ & 0.9800 \\
\hline C59-H59A & 0.9800 & $\mathrm{C} 28-\mathrm{H} 28 \mathrm{~B}$ & 0.9800 \\
\hline $\mathrm{C} 56-\mathrm{C} 58$ & $1.531(6)$ & $\mathrm{C} 28-\mathrm{H} 28 \mathrm{C}$ & 0.9800 \\
\hline $\mathrm{C} 2-\mathrm{Ru} 1-\mathrm{N} 5$ & $90.54(14)$ & $\mathrm{C} 59-\mathrm{C} 53-\mathrm{Ru} 1$ & $128.4(3)$ \\
\hline $\mathrm{C} 2-\mathrm{Ru} 1-\mathrm{N} 3$ & $76.59(15)$ & $\mathrm{C} 53-\mathrm{C} 54-\mathrm{C} 55$ & $121.3(4)$ \\
\hline $\mathrm{N} 5-\mathrm{Ru} 1-\mathrm{N} 3$ & $89.02(13)$ & $\mathrm{C} 53-\mathrm{C} 54-\mathrm{Ru} 1$ & $72.3(2)$ \\
\hline $\mathrm{C} 2-\mathrm{Ru} 1-\mathrm{C} 54$ & $93.05(15)$ & $\mathrm{C} 55-\mathrm{C} 54-\mathrm{Ru} 1$ & $71.7(2)$ \\
\hline $\mathrm{N} 5-\mathrm{Ru} 1-\mathrm{C} 54$ & $152.34(14)$ & $\mathrm{C} 53-\mathrm{C} 54-\mathrm{H} 54$ & 119.4 \\
\hline $\mathrm{N} 3-\mathrm{Ru} 1-\mathrm{C} 54$ & $118.48(14)$ & $\mathrm{C} 55-\mathrm{C} 54-\mathrm{H} 54$ & 119.4 \\
\hline $\mathrm{C} 2-\mathrm{Ru} 1-\mathrm{C} 55$ & $96.00(16)$ & $\mathrm{Ru} 1-\mathrm{C} 54-\mathrm{H} 54$ & 129.0 \\
\hline $\mathrm{N} 5-\mathrm{Ru} 1-\mathrm{C} 55$ & $115.03(14)$ & $\mathrm{C} 50-\mathrm{C} 55-\mathrm{C} 54$ & $120.5(4)$ \\
\hline $\mathrm{N} 3-\mathrm{Ru} 1-\mathrm{C} 55$ & $155.13(14)$ & $\mathrm{C} 50-\mathrm{C} 55-\mathrm{Ru} 1$ & $72.7(2)$ \\
\hline $\mathrm{C} 54-\mathrm{Ru} 1-\mathrm{C} 55$ & $37.32(15)$ & $\mathrm{C} 54-\mathrm{C} 55-\mathrm{Ru} 1$ & $71.0(2)$ \\
\hline $\mathrm{C} 2-\mathrm{Ru} 1-\mathrm{C} 53$ & $116.11(15)$ & $\mathrm{C} 50-\mathrm{C} 55-\mathrm{H} 55$ & 119.8 \\
\hline $\mathrm{N} 5-\mathrm{Ru} 1-\mathrm{C} 53$ & $153.20(14)$ & C54-C55-H55 & 119.8 \\
\hline N3-Ru1-C53 & $94.33(14)$ & $\mathrm{Ru} 1-\mathrm{C} 55-\mathrm{H} 55$ & 128.8 \\
\hline $\mathrm{C} 54-\mathrm{Ru} 1-\mathrm{C} 53$ & $37.11(14)$ & $\mathrm{C} 53-\mathrm{C} 59-\mathrm{H} 59 \mathrm{C}$ & 109.5 \\
\hline
\end{tabular}




$$
\begin{aligned}
& \text { C55-Ru1-C53 } \\
& \text { C2-Ru1-C50 } \\
& \text { N5-Ru1-C50 } \\
& \text { N3-Ru1-C50 } \\
& \text { C54-Ru1-C50 } \\
& \text { C55-Ru1-C50 } \\
& \text { C53-Ru1-C50 } \\
& \text { C2-Ru1-C52 } \\
& \text { N5-Ru1-C52 } \\
& \text { N3-Ru1-C52 } \\
& \text { C54-Ru1-C52 } \\
& \text { C55-Ru1-C52 } \\
& \text { C53-Ru1-C52 } \\
& \text { C50-Ru1-C52 } \\
& \text { C2-Ru1-C51 } \\
& \text { N5-Ru1-C51 } \\
& \text { N3-Ru1-C51 } \\
& \text { C54-Ru1-C51 } \\
& \text { C55-Ru1-C51 } \\
& \text { C53-Ru1-C51 } \\
& \text { C50-Ru1-C51 } \\
& \text { C52-Ru1-C51 } \\
& \text { F5-Sb1-F6 } \\
& \text { F5-Sb1-F4 } \\
& \text { F6-Sb1-F4 } \\
& \text { F5-Sb1-F1 } \\
& \text { F6-Sb1-F1 } \\
& \text { F4-Sb1-F1 } \\
& \text { F5-Sb1-F3 } \\
& \text { F6-Sb1-F3 } \\
& \text { F4-Sb1-F3 } \\
& \text { F1-Sb1-F3 } \\
& \text { F5-Sb1-F2 } \\
& \text { F6-Sb1-F2 } \\
& \text { F4-Sb1-F2 } \\
& \text { F1-Sb1-F2 } \\
& \text { F3-Sb1-F2 } \\
& \text { F10-Sb2-F12 } \\
& \text { F10-Sb2-F8 } \\
& \text { F12-Sb2-F8 } \\
& \text { F10-Sb2-F11 } \\
& \text { F12-Sb2-F11 } \\
& \text { F8-Sb2-F11 } \\
& \text { F10-Sb2-F7 } \\
& \text { F12-Sb2-F7 } \\
& \text { F8-Sb2-F7 } \\
& \text { F11-Sb2-F7 } \\
& \text { F10-Sb2-F9 }
\end{aligned}
$$

$$
67.31(15)
$$

\begin{tabular}{|c|c|}
\hline $\mathrm{C} 53-\mathrm{C} 59-\mathrm{H} 59 \mathrm{~B}$ & 109.5 \\
\hline $\mathrm{H} 59 \mathrm{C}-\mathrm{C} 59-\mathrm{H} 59 \mathrm{~B}$ & 109.5 \\
\hline $\mathrm{C} 53-\mathrm{C} 59-\mathrm{H} 59 \mathrm{~A}$ & 109.5 \\
\hline $\mathrm{H} 59 \mathrm{C}-\mathrm{C} 59-\mathrm{H} 59 \mathrm{~A}$ & 109.5 \\
\hline $\mathrm{H} 59 \mathrm{~B}-\mathrm{C} 59-\mathrm{H} 59 \mathrm{~A}$ & 109.5 \\
\hline $\mathrm{C} 50-\mathrm{C} 56-\mathrm{C} 58$ & $113.9(4)$ \\
\hline $\mathrm{C} 50-\mathrm{C} 56-\mathrm{C} 57$ & $107.7(4)$ \\
\hline $\mathrm{C} 58-\mathrm{C} 56-\mathrm{C} 57$ & $112.1(4)$ \\
\hline $\mathrm{C} 50-\mathrm{C} 56-\mathrm{H} 56$ & 107.6 \\
\hline $\mathrm{C} 58-\mathrm{C} 56-\mathrm{H} 56$ & 107.6 \\
\hline $\mathrm{C} 57-\mathrm{C} 56-\mathrm{H} 56$ & 107.6 \\
\hline $\mathrm{C} 56-\mathrm{C} 57-\mathrm{H} 57 \mathrm{~A}$ & 109.5 \\
\hline $\mathrm{C} 56-\mathrm{C} 57-\mathrm{H} 57 \mathrm{~B}$ & 109.5 \\
\hline $\mathrm{H} 57 \mathrm{~A}-\mathrm{C} 57-\mathrm{H} 57 \mathrm{~B}$ & 109.5 \\
\hline $\mathrm{C} 56-\mathrm{C} 57-\mathrm{H} 57 \mathrm{C}$ & 109.5 \\
\hline $\mathrm{H} 57 \mathrm{~A}-\mathrm{C} 57-\mathrm{H} 57 \mathrm{C}$ & 109.5 \\
\hline $\mathrm{H} 57 \mathrm{~B}-\mathrm{C} 57-\mathrm{H} 57 \mathrm{C}$ & 109.5 \\
\hline $\mathrm{C} 56-\mathrm{C} 58-\mathrm{H} 58 \mathrm{~A}$ & 109.5 \\
\hline $\mathrm{C} 56-\mathrm{C} 58-\mathrm{H} 58 \mathrm{C}$ & 109.5 \\
\hline $\mathrm{H} 58 \mathrm{~A}-\mathrm{C} 58-\mathrm{H} 58 \mathrm{C}$ & 109.5 \\
\hline $\mathrm{C} 56-\mathrm{C} 58-\mathrm{H} 58 \mathrm{~B}$ & 109.5 \\
\hline $\mathrm{H} 58 \mathrm{~A}-\mathrm{C} 58-\mathrm{H} 58 \mathrm{~B}$ & 109.5 \\
\hline $\mathrm{H} 58 \mathrm{C}-\mathrm{C} 58-\mathrm{H} 58 \mathrm{~B}$ & 109.5 \\
\hline $\mathrm{N} 1-\mathrm{C} 2-\mathrm{C} 3$ & $107.6(3)$ \\
\hline $\mathrm{N} 1-\mathrm{C} 2-\mathrm{Ru} 1$ & $117.3(3)$ \\
\hline $\mathrm{C} 3-\mathrm{C} 2-\mathrm{Ru} 1$ & $135.1(3)$ \\
\hline $\mathrm{C} 2-\mathrm{C} 3-\mathrm{C} 4$ & $104.4(3)$ \\
\hline $\mathrm{C} 2-\mathrm{C} 3-\mathrm{H} 3 \mathrm{~A}$ & 110.9 \\
\hline $\mathrm{C} 4-\mathrm{C} 3-\mathrm{H} 3 \mathrm{~A}$ & 110.9 \\
\hline $\mathrm{C} 2-\mathrm{C} 3-\mathrm{H} 3 \mathrm{~B}$ & 110.9 \\
\hline $\mathrm{C} 4-\mathrm{C} 3-\mathrm{H} 3 \mathrm{~B}$ & 110.9 \\
\hline $\mathrm{H} 3 \mathrm{~A}-\mathrm{C} 3-\mathrm{H} 3 \mathrm{~B}$ & 108.9 \\
\hline $\mathrm{C} 5-\mathrm{C} 4-\mathrm{C} 9$ & $119.0(4)$ \\
\hline $\mathrm{C} 5-\mathrm{C} 4-\mathrm{C} 3$ & $132.5(4)$ \\
\hline $\mathrm{C} 9-\mathrm{C} 4-\mathrm{C} 3$ & $108.5(3)$ \\
\hline $\mathrm{C} 8-\mathrm{C} 9-\mathrm{C} 4$ & $123.8(4)$ \\
\hline $\mathrm{C} 8-\mathrm{C} 9-\mathrm{N} 1$ & $129.6(4)$ \\
\hline $\mathrm{C} 4-\mathrm{C} 9-\mathrm{N} 1$ & $106.7(3)$ \\
\hline $\mathrm{C} 9-\mathrm{C} 8-\mathrm{C} 7$ & $116.6(4)$ \\
\hline $\mathrm{C} 9-\mathrm{C} 8-\mathrm{H} 8$ & 121.7 \\
\hline $\mathrm{C} 7-\mathrm{C} 8-\mathrm{H} 8$ & 121.7 \\
\hline $\mathrm{C} 6-\mathrm{C} 7-\mathrm{C} 8$ & $120.3(4)$ \\
\hline $\mathrm{C} 6-\mathrm{C} 7-\mathrm{H} 7$ & 119.8 \\
\hline $\mathrm{C} 8-\mathrm{C} 7-\mathrm{H} 7$ & 119.8 \\
\hline $\mathrm{C} 5-\mathrm{C} 6-\mathrm{C} 7$ & $121.5(4)$ \\
\hline $\mathrm{C} 5-\mathrm{C} 6-\mathrm{H} 6$ & 119.2 \\
\hline $\mathrm{C} 7-\mathrm{C} 6-\mathrm{H} 6$ & 119.2 \\
\hline $\mathrm{C} 4-\mathrm{C} 5-\mathrm{C} 6$ & $118.7(4)$ \\
\hline
\end{tabular}$$
123.14(16)
$$$$
88.31(14)
$$$$
160.11(14)
$$$$
66.88(15)
$$$$
36.89(14)
$$$$
79.57(15)
$$$$
152.89(15)
$$$$
116.18(14)
$$$$
98.24(14)
$$$$
65.67(15)
$$$$
77.44(15)
$$$$
37.03(14)
$$$$
65.55(15)
$$$$
160.05(16)
$$$$
90.72(14)
$$$$
123.34(14)
$$$$
76.96(15)
$$$$
65.56(15)
$$$$
65.32(15)
$$$$
37.03(15)
$$$$
34.79(15)
$$$$
91.35(15)
$$$$
90.48(13)
$$$$
178.08(15)
$$$$
178.35(14)
$$$$
89.71(14)
$$$$
88.45(12)
$$$$
89.91(12)
$$$$
90.98(12)
$$$$
89.61(11)
$$$$
91.34(12)
$$$$
88.79 \text { (11) }
$$$$
88.99(11)
$$$$
90.46(10)
$$$$
89.96 \text { (12) }
$$$$
178.70(12)
$$$$
91.0 \text { (2) }
$$$$
90.89 \text { (17) }
$$$$
178.02(18)
$$$$
178.52(18)
$$$$
90.30 \text { (19) }
$$$$
87.86 \text { (17) }
$$$$
91.56(15)
$$$$
89.85 \text { (14) }
$$$$
89.37 \text { (13) }
$$$$
89.24 \text { (15) }
$$$$
89.76 \text { (14) }
$$ 


\begin{tabular}{|c|c|c|c|}
\hline $\mathrm{F} 12-\mathrm{Sb} 2-\mathrm{F} 9$ & $89.97(14)$ & $\mathrm{C} 4-\mathrm{C} 5-\mathrm{H} 5$ & 120.6 \\
\hline $\mathrm{F} 8-\mathrm{Sb} 2-\mathrm{F} 9$ & $90.77(12)$ & $\mathrm{C} 6-\mathrm{C} 5-\mathrm{H} 5$ & 120.6 \\
\hline $\mathrm{F} 11-\mathrm{Sb} 2-\mathrm{F} 9$ & $89.45(14)$ & $\mathrm{C} 2-\mathrm{N} 1-\mathrm{C} 10$ & $117.4(3)$ \\
\hline $\mathrm{F} 7-\mathrm{Sb} 2-\mathrm{F} 9$ & $178.67(15)$ & $\mathrm{C} 2-\mathrm{N} 1-\mathrm{C} 9$ & $112.8(3)$ \\
\hline $\mathrm{C} 27-\mathrm{N} 5-\mathrm{Ru} 1$ & $170.4(3)$ & $\mathrm{C} 10-\mathrm{N} 1-\mathrm{C} 9$ & $129.7(3)$ \\
\hline $\mathrm{C} 55-\mathrm{C} 50-\mathrm{C} 51$ & $117.9(4)$ & $\mathrm{N} 2-\mathrm{C} 10-\mathrm{N} 3$ & $127.8(4)$ \\
\hline $\mathrm{C} 55-\mathrm{C} 50-\mathrm{C} 56$ & $123.1(4)$ & $\mathrm{N} 2-\mathrm{C} 10-\mathrm{N} 1$ & $120.4(4)$ \\
\hline $\mathrm{C} 51-\mathrm{C} 50-\mathrm{C} 56$ & $118.9(4)$ & $\mathrm{N} 3-\mathrm{C} 10-\mathrm{N} 1$ & $111.8(3)$ \\
\hline $\mathrm{C} 55-\mathrm{C} 50-\mathrm{Ru} 1$ & $70.4(2)$ & $\mathrm{C} 10-\mathrm{N} 2-\mathrm{C} 11$ & $114.9(4)$ \\
\hline $\mathrm{C} 51-\mathrm{C} 50-\mathrm{Ru} 1$ & $73.8(2)$ & $\mathrm{N} 2-\mathrm{C} 11-\mathrm{C} 12$ & $123.1(4)$ \\
\hline $\mathrm{C} 56-\mathrm{C} 50-\mathrm{Ru} 1$ & $129.7(3)$ & $\mathrm{N} 2-\mathrm{C} 11-\mathrm{H} 11$ & 118.5 \\
\hline $\mathrm{C} 52-\mathrm{C} 51-\mathrm{C} 50$ & $121.4(4)$ & $\mathrm{C} 12-\mathrm{C} 11-\mathrm{H} 11$ & 118.5 \\
\hline $\mathrm{C} 52-\mathrm{C} 51-\mathrm{Ru} 1$ & $72.5(2)$ & $\mathrm{C} 11-\mathrm{C} 12-\mathrm{C} 13$ & $117.1(4)$ \\
\hline $\mathrm{C} 50-\mathrm{C} 51-\mathrm{Ru} 1$ & $69.2(2)$ & $\mathrm{C} 11-\mathrm{C} 12-\mathrm{H} 12$ & 121.4 \\
\hline $\mathrm{C} 52-\mathrm{C} 51-\mathrm{H} 51$ & 119.3 & $\mathrm{C} 13-\mathrm{C} 12-\mathrm{H} 12$ & 121.4 \\
\hline $\mathrm{C} 50-\mathrm{C} 51-\mathrm{H} 51$ & 119.3 & $\mathrm{~N} 3-\mathrm{C} 13-\mathrm{C} 12$ & $121.4(4)$ \\
\hline $\mathrm{Ru} 1-\mathrm{C} 51-\mathrm{H} 51$ & 132.0 & $\mathrm{~N} 3-\mathrm{C} 13-\mathrm{H} 13$ & 119.3 \\
\hline $\mathrm{C} 51-\mathrm{C} 52-\mathrm{C} 53$ & $120.5(4)$ & $\mathrm{C} 12-\mathrm{C} 13-\mathrm{H} 13$ & 119.3 \\
\hline $\mathrm{C} 51-\mathrm{C} 52-\mathrm{Ru} 1$ & $72.7(2)$ & $\mathrm{C} 13-\mathrm{N} 3-\mathrm{C} 10$ & $115.6(3)$ \\
\hline $\mathrm{C} 53-\mathrm{C} 52-\mathrm{Ru} 1$ & $68.6(2)$ & $\mathrm{C} 13-\mathrm{N} 3-\mathrm{Ru} 1$ & $127.6(3)$ \\
\hline $\mathrm{C} 51-\mathrm{C} 52-\mathrm{H} 52$ & 119.7 & $\mathrm{C} 10-\mathrm{N} 3-\mathrm{Ru} 1$ & $116.8(3)$ \\
\hline $\mathrm{C} 53-\mathrm{C} 52-\mathrm{H} 52$ & 119.7 & $\mathrm{~N} 5-\mathrm{C} 27-\mathrm{C} 28$ & $178.9(5)$ \\
\hline $\mathrm{Ru} 1-\mathrm{C} 52-\mathrm{H} 52$ & 131.8 & $\mathrm{C} 27-\mathrm{C} 28-\mathrm{H} 28 \mathrm{~A}$ & 109.5 \\
\hline $\mathrm{C} 54-\mathrm{C} 53-\mathrm{C} 52$ & $118.2(4)$ & $\mathrm{C} 27-\mathrm{C} 28-\mathrm{H} 28 \mathrm{~B}$ & 109.5 \\
\hline $\mathrm{C} 54-\mathrm{C} 53-\mathrm{C} 59$ & $122.0(4)$ & $\mathrm{H} 28 \mathrm{~A}-\mathrm{C} 28-\mathrm{H} 28 \mathrm{~B}$ & 109.5 \\
\hline $\mathrm{C} 52-\mathrm{C} 53-\mathrm{C} 59$ & $119.8(4)$ & $\mathrm{C} 27-\mathrm{C} 28-\mathrm{H} 28 \mathrm{C}$ & 109.5 \\
\hline $\mathrm{C} 54-\mathrm{C} 53-\mathrm{Ru} 1$ & $70.6(2)$ & $\mathrm{H} 28 \mathrm{~A}-\mathrm{C} 28-\mathrm{H} 28 \mathrm{C}$ & 109.5 \\
\hline $\mathrm{C} 52-\mathrm{C} 53-\mathrm{Ru} 1$ & $74.3(2)$ & $\mathrm{H} 28 \mathrm{~B}-\mathrm{C} 28-\mathrm{H} 28 \mathrm{C}$ & 109.5 \\
\hline
\end{tabular}

Hydrogen-bond geometry $\left(\AA,{ }^{\circ}\right)$

\begin{tabular}{lllll}
\hline$D-\mathrm{H} \cdots A$ & $D-\mathrm{H}$ & $\mathrm{H} \cdots A$ & $D \cdots A$ & $D-\mathrm{H} \cdots A$ \\
\hline $\mathrm{C} 11-\mathrm{H} 11 \cdots \mathrm{F} 7^{\mathrm{i}}$ & 0.95 & 2.54 & $3.398(6)$ & 151 \\
$\mathrm{C} 12-\mathrm{H} 12 \cdots \mathrm{F} 1$ & 0.95 & 2.39 & $3.157(5)$ & 138 \\
$\mathrm{C} 13-\mathrm{H} 13 \cdots \mathrm{F} 2$ & 0.95 & 2.30 & $3.229(5)$ & 167 \\
$\mathrm{C} 51-\mathrm{H} 51 \cdots \mathrm{F} 11^{\mathrm{ii}}$ & 0.95 & 2.54 & $3.485(6)$ & 174 \\
$\mathrm{C} 52-\mathrm{H} 52 \cdots \mathrm{F} 2$ & 0.95 & 2.50 & $3.337(5)$ & 147 \\
$\mathrm{C} 54-\mathrm{H} 54 \cdots \mathrm{F} 5$ iii & 0.95 & 2.26 & $3.110(5)$ & 148 \\
$\mathrm{C} 59-\mathrm{H} 59 B \cdots \mathrm{F} 6$ & 0.98 & 2.32 & $3.253(6)$ & 158 \\
$\mathrm{C} 59-\mathrm{H} 59 C \cdots \mathrm{F} 5^{\mathrm{iii}}$ & 0.98 & 2.45 & $3.270(6)$ & 141
\end{tabular}

Symmetry codes: (i) $x,-y+1 / 2, z+1 / 2$; (ii) $-x+2, y-1 / 2,-z+3 / 2$; (iii) $-x+3 / 2, y+1 / 2, z$. 